Service social

\title{
La mesure du soutien social
}

\section{Line Beauregard et Serge Dumont}

Volume 45, numéro 3, 1996

Santé

URI : https://id.erudit.org/iderudit/706737ar

DOI : https://doi.org/10.7202/706737ar

Aller au sommaire du numéro

Éditeur(s)

École de service social de l'Université Laval

ISSN

1708-1734 (numérique)

Découvrir la revue

Citer cet article

Beauregard, L. \& Dumont, S. (1996). La mesure du soutien social. Service social, 45(3), 55-76. https://doi.org/10.7202/706737ar
Résumé de l'article

Dans le présent article, les auteurs examinent le concept de soutien social en apportant une attention particulière à sa définition et à sa mesure. Par une recension des écrits, les auteurs visent à clarifier la notion de soutien social et à identifier des instruments de mesure qui correspondent aux diverses définitions proposées dans les articles consultés. Cette recension montre que le soutien social est composé de trois dimensions, soit les ressources du réseau de soutien, les comportements de soutien et l'appréciation subjective de soutien. Après avoir décrit neuf instruments qui mesurent une ou plusieurs de ces dimensions, l'article se termine par une discussion qui porte sur des recommandations liées au choix d'un instrument pour mesurer le soutien social. 


\section{La mesure du soutien social}

Line BEAUREGARD

Étudiante au doctorat en service social Membre étudiante du Centre de recherche sur les services communautaires Université Laval

Serge DuMONT

Professeur adjoint École de service social Université Laval

\section{INTRODUCTION}

Depuis les années 1970, nous constatons un intérêt croissant de la recherche psychosociale pour le concept de soutien social, perçu comme une variable déterminante en santé physique et mentale (Bozzini et Tessier, 1985). Cependant, certains problèmes se présentent, notamment en ce qui concerne la définition conceptuelle du soutien social et les limites inhérentes aux instruments élaborés à ce jour pour en permettre la mesure.

D'entrée de jeu, il est utile de signaler que plusieurs auteurs relèvent un manque de consensus entre les chercheurs quant à la définition du concept de soutien social (Ducharme, Stevens et 
Rowat, 1994; Gottlieb, 1983, dans Barrera, 1986; Heitzmann et Kaplan, 1988 ; O’Reilly, 1988 ; Sarason et al., 1987 ; Tardy, 1985 ; Winemiller et al., 1993). On remarque également que le concept de soutien social n'est pas toujours défini de façon précise (Barrera, 1986; Bruhn, 1991; House et al., 1985 ; Vaux, 1988, 1992; Winemiller et al., 1993).

Ces difficultés conceptuelles ont inévitablement des conséquences sur le plan de la mesure du soutien social. Ainsi, l'absence de consensus sur la définition conceptuelle fait en sorte qu'il existe de nombreux instruments de mesure, ces derniers étant souvent élaborés par les chercheurs pour répondre aux besoins de leur propre recherche (Heitzmann et Kaplan, 1988; Tardy, 1985 ; Vaux, 1992; Weinert et Tilden, 1990; Winemiller et al., 1993). De plus, plusieurs instruments de mesure du soutien social présentent des limites sur le plan métrologique : la fidélité et la validité sont tantôt faibles, tantôt inconnues, ou encore l'instrument n'a pas été soumis à des tests ayant pour but d'évaluer leur fiabilité (Bruhn, 1991; Cohen et Syme, 1985; Heitzmann et Kaplan, 1988 ; O’Reilly, 1988 ; Rock et al., 1984 ; Tardy, 1985 ; Vaux, 1992; Weinert et Tilden, 1990; Winemiller et al., 1993). L'utilisation d'instruments inadéquats biaise les résultats des recherches et contribue sans doute à produire une certaine inconsistance dans leurs conclusions (Bruhn, 1991; Cohen et McKay, 1984, dans Barrera, 1986; Heitzmann et Kaplan, 1988). Tardy (1985) mentionne également que les référents conceptuels sous-jacents à certains instruments ne sont pas mentionnés par les auteurs; ils doivent être inférés, ce qui contribue à générer de mauvaises interprétations et des généralisations incertaines.

Il ne semble donc pas exister de consensus clairement établi autour de la définition et de l'opérationnalisation du concept de soutien social (Blanchard et al., 1995 ; Buchanan, 1995). Toutefois, malgré ces difficultés, l'importance de poursuivre les efforts de recherche sur le soutien social, notamment pour mieux en comprendre le processus, est d'emblée reconnue. Ces efforts devraient conduire à une clarification du concept et à un raffinement de la mesure.

Au moyen d'une analyse descriptive, le présent article se propose d'identifier les principales définitions conceptuelles du soutien social recensées dans la littérature, de même que les principaux instruments de mesure construits sur la base de ces définitions. 


\section{PRINCIPALES DÉFINITIONS CONCEPTUELLES DU SOUTIEN SOCIAL}

Les différentes façons de concevoir le soutien social introduisent des terminologies différentes pour désigner parfois le même concept (voir tableau 1). Malgré la diversité de points de vue, il semble exister, dans la communauté scientifique, un consensus selon lequel le soutien social est un concept multidimensionnel. Certains auteurs ont proposé des catégories pour décrire ces multiples dimensions afin de classifier les instruments qui existent. Ainsi, Barrera (1986) ainsi que Streeter et Franklin (1992) présentent trois dimensions du soutien social, soit: l'intégration

\section{Tableau 1}

Dimensions du soutien social selon les auteurs et la terminologie utilisée

\begin{tabular}{|c|c|c|}
\hline DIMENSIONS & AUTEURS & TERMINOLOGIE \\
\hline \multirow{3}{*}{ Le réseau de soutien } & $\begin{array}{l}\text { Barrera (1986) } \\
\text { Streeter et Franklin (1992) }\end{array}$ & $\begin{array}{l}\text { L'intégration sociale } \\
\text { (social embeddedness) }\end{array}$ \\
\hline & $\begin{array}{l}\text { Vaux (1988, 1992) } \\
\text { Vaux et al. (1986) }\end{array}$ & $\begin{array}{l}\text { Les ressources du réseau } \\
\text { de soutien } \\
\text { (support network resources) }\end{array}$ \\
\hline & $\begin{array}{l}\text { Pierce, Sarason et Sarason } \\
\text { (1996) }\end{array}$ & $\begin{array}{l}\text { Le réseau de soutien } \\
\text { (supportive network) }\end{array}$ \\
\hline \multirow{3}{*}{$\begin{array}{l}\text { Les comportements } \\
\text { de soutien }\end{array}$} & $\begin{array}{l}\text { Barrera (1986) } \\
\text { Streeter et Franklin (1992) }\end{array}$ & $\begin{array}{l}\text { Le soutien reçu } \\
\text { (enacted support) }\end{array}$ \\
\hline & $\begin{array}{l}\text { Vaux }(1988,1992) \\
\text { Vaux et al. }(1986)\end{array}$ & $\begin{array}{l}\text { Les comportements } \\
\text { de soutien } \\
\text { (supportive behavior) }\end{array}$ \\
\hline & $\begin{array}{l}\text { Pierce, Sarason } \\
\text { et Sarason (1996) }\end{array}$ & $\begin{array}{l}\text { Les relations de soutien } \\
\text { (supportive relationships) }\end{array}$ \\
\hline \multirow{3}{*}{$\begin{array}{l}\text { L'appréciation } \\
\text { subjective du soutien }\end{array}$} & $\begin{array}{l}\text { Barrera (1986) } \\
\text { Streeter et Franklin (1992) }\end{array}$ & $\begin{array}{l}\text { La perception } \\
\text { du soutien social } \\
\text { (perceived social support) }\end{array}$ \\
\hline & $\begin{array}{l}\text { Vaux }(1988,1992) \\
\text { Vaux et al. }(1986)\end{array}$ & $\begin{array}{l}\text { L'appréciation du soutien } \\
\text { (support appraisals) }\end{array}$ \\
\hline & $\begin{array}{l}\text { Pierce, Sarason et Sarason } \\
\text { (1996) }\end{array}$ & $\begin{array}{l}\text { La perception } \\
\text { du soutien social } \\
\text { (perceived social support) }\end{array}$ \\
\hline
\end{tabular}


sociale (social embeddedness), le soutien reçu (enacted support) et, enfin, la perception du soutien social (perceived social support). D'autres auteurs, tels que Vaux (1988 et 1992), Vaux et al. (1986), proposent les trois dimensions suivantes: les ressources du réseau de soutien (support network resources), les comportements de soutien (supportive behavior) et, enfin, l'appréciation du soutien (support appraisals). Plus récemment, Pierce, Sarason et Sarason (1996) ont suggéré, eux aussi, trois dimensions au soutien social qui s'apparentent à la proposition de Vaux et de ses collaborateurs. Il s'agit du réseau de soutien (supportive network), des relations de soutien (supportive relationships) et de la perception du soutien social (perceived social support). Plusieurs termes sont donc évoqués pour identifier les différentes dimensions du soutien social et le tableau 1 présente une synthèse de ces différents concepts, approfondis par la suite.

\section{Le réseau de soutien}

Vaux (1988 et 1992) définit le réseau de soutien de la façon suivante: "Le réseau de soutien est un sous-ensemble du réseau social, vers qui la personne se tourne (ou pourrait se tourner) pour obtenir de l'aide...» (Vaux, 1988, p. 28, traduction libre). Lorsqu'on parle des ressources du réseau de soutien, on fait référence à la grandeur du réseau de soutien, à sa structure ou encore à ses caractéristiques relationnelles (Vaux et al., 1986). Barrera (1986) ainsi que Streeter et Franklin (1992) recourent à l'expression "intégration sociale» (social embeddedness) pour signifier les liens ou contacts d'un individu avec les autres personnes significatives de son environnement. L'intégration sociale serait associée au sentiment d'appartenance à la communauté (Streeter et Franklin, 1992).

Les instruments qui mesurent les ressources du réseau identifient les rapports qui relient la personne à sa famille, à ses amis, à ses pairs. Ces rapports sont vus comme un indicateur des ressources sociales qui, lors d'une crise, sont susceptibles d'offrir du soutien (Streeter et Franklin, 1992). Les mesures du réseau évaluent le nombre d'individus et de liens à l'intérieur du réseau (Rock et al., 1984). Selon Barrera (1986), il existe deux approches pour mesurer les ressources du réseau (ou l'intégration sociale, pour utiliser l'expression de cet auteur). Une première approche consiste à utiliser des indicateurs de la présence de liens sociaux tels que le statut marital, la présence de fratrie plus âgée, la participation à des organisations de la communauté et autres 
types d'indicateurs. Ces indicateurs sont utilisés avec l'hypothèse que les liens sociaux disponibles peuvent fournir du soutien lors de crises. Une deuxième approche consiste à analyser le réseau social. Cette forme d'analyse implique souvent une procédure structurée pour identifier les individus qui ont des relations importantes avec la personne. L'accent est alors mis sur les propriétés structurelles du réseau, telles que la densité, la complexité, l'accessibilité et quelques autres caractéristiques.

Une critique faite à l'endroit des instruments qui mesurent les ressources du réseau est liée au fait que ceux-ci ne permettent pas de démontrer comment ces ressources apportent du soutien social et influencent l'état de la personne (Barrera, 1986). De plus, on ne peut prétendre que les relations identifiées par une analyse de réseau sont automatiquement des relations de soutien (Wellman, 1981). Cependant, les instruments de mesure qui identifient les membres d'un réseau selon le type de soutien apporté (ex. : conseils, information, soutien émotionnel, etc.) ne se limitent pas à une quantification des liens sociaux; ils offrent également la possibilité de documenter le contenu de ces liens (Barrera, 1981; Norbeck, Lindsey et Carrieri, 1981).

\section{Les comportements de soutien}

Le soutien social est aussi conceptualisé comme étant l'ensemble des actions ou des comportements qui fournissent effectivement de l'aide à la personne (Barrera, 1986). Il s'agit d'un soutien actif (en opposition à un soutien potentiel) composé d'actions ou de comportements spécifiques effectués par les autres et considérés comme une expression manifeste de soutien ou d'assistance. Le soutien reçu fait donc référence à l'utilisation actuelle des ressources du soutien social (Tardy, 1985). On le présente aussi comme étant une description comportementale du soutien. Les comportements de soutien peuvent comprendre l'écoute, l'expression des inquiétudes, le prêt d'argent, l'aide pour réaliser une tâche, avoir l'opinion des autres, montrer de l'affection, etc.

Les instruments générés dans cette perspective sont destinés à inventorier les comportements spécifiques d'aide survenant dans un contexte de relations (Vaux, 1992). Néanmoins, la mesure de ces comportements d'aide repose sur un rappel d'expériences antérieures plutôt que d'observations actuelles, ce qui, bien sûr, introduit une certaine limite (Streeter et Franklin, 1992). 


\section{L'appréciation subjective de soutien}

L'appréciation subjective de soutien fait référence à l'évaluation cognitive d'une personne à propos du soutien qu'elle estime recevoir d'autrui (Barrera, 1986; Streeter et Franklin, 1992). Cette appréciation regroupe plusieurs dimensions, telles que le sentiment d'avoir suffisamment de soutien, la satisfaction à l'égard du soutien reçu, la perception selon laquelle les besoins de soutien sont comblés, la perception de la disponibilité et de l'adéquation du soutien et, enfin, la confiance que le soutien sera disponible en cas de besoin (Barrera, 1986; Streeter et Franklin, 1992; Vaux, 1992). Tout comme pour les deux autres concepts présentés plus haut, l'appréciation subjective peut porter sur divers types de soutien, tels que l'aide matérielle, informationnelle ou émotionnelle. Il est postulé que ce ne sont pas tous les rapports d'une personne avec son environnement qui fournissent du soutien. Le soutien serait lié à une expérience personnelle plutôt qu'à un ensemble de circonstances objectives (Buchanan, 1995). Cela justifie le fait de mesurer la perception de soutien. L'étude de Tracy et Abell (1994) confirme, par ailleurs, que l'information à propos des caractéristiques structurelles, telle la grandeur du réseau social, ne peut à elle seule fournir une évaluation de la qualité et de la quantité du soutien social. De très petits réseaux peuvent s'avérer supportants, alors que de grands réseaux, au contraire, peuvent ne pas apporter de soutien. Les instruments destinés à évaluer le réseau social devraient donc comporter des questions à propos de l'appréciation subjective de soutien (Tracy et Abell, 1994).

D'autre part, certains auteurs estiment que la mesure du soutien perçu peut être affectée par des variables susceptibles d'avoir un effet potentiellement confondant, tels le stress ou la détresse psychologique (Barrera, 1986; Tousignant, 1988).

\section{TYPES ET SOURCES DE SOUTIEN}

Le présent article a mis en relief que le soutien social se compose de trois dimensions, soit un réseau qui comporte une certaine quantité de ressources, un ensemble de comportements ou de gestes concrets visant à apporter du soutien et, enfin, le soutien perçu, c'est-à-dire l'appréciation subjective du soutien. Chacune de ces dimensions se caractérise selon un type et une source. Le type de soutien fait référence à la nature des activités exercées 
dans le but d'apporter une aide particulière, alors que la source indique la ou les personnes qui fournissent ce soutien.

\section{Types de soutien}

Il est important de tenir compte des types de soutien, car le contexte spécifique de l'aide joue un rôle dans son efficacité; ainsi, selon le contexte de la situation, certains types de soutien peuvent être inappropriés (Cohen et Wills, 1985). Des auteurs ont élaboré des typologies qui proposent des catégorisations élaborées selon le type de soutien. Le tableau 2 de la page suivante présente une synthèse de certaines de ces typologies.

L'analyse du tableau 2 permet d'entrevoir une distinction fondamentale voulant que les différents types de soutien soient associés soit à de l'aide tangible (ex.: aide financière), soit à de l'aide non tangible (ex.: soutien émotionnel). Cette distinction demeure toutefois relative, puisqu'un même geste peut être associé à plus d'un type de soutien. Par exemple, prêter de l'argent ou fournir un moyen de transport procurent un soutien de type instrumental, mais ils ont aussi le potentiel de donner un soutien émotionnel.

\section{Sources de soutien}

Selon Vaux (1992), la source ou la provenance du soutien social revêt une importance particulière, notamment lorsqu'il est question de sa mesure. Dans la même perspective, Streeter et Franklin (1992) insistent sur l'importance de faire la distinction entre les sources formelles et les sources informelles. Les sources informelles sont le soutien des amis, de la famille, etc., tandis que les sources formelles de soutien proviennent des organisations formelles (ex.: services gouvernementaux, agences privées). Les premières sont beaucoup moins structurées que les deuxièmes. Ainsi, le soutien formel est synonyme d'organisation bureaucratique, de spécialisation des fonctions des intervenants, de procédures et de règles, de critères explicites pour l'évaluation des besoins et l'admissibilité aux services, etc. Le soutien informel n'est pas soumis à de telles contraintes; il s'actualise avec davantage de souplesse (Streeter et Franklin, 1992).

Richman, Rosenfeld et Hardy (1993) établissent un lien entre le type de soutien et sa source. Selon eux, certains types de soutien requièrent une expertise spécifique (appréciation du 
travail réalisé dans un emploi rémunéré), alors que d'autres n'en demandent pas (ex. : l'écoute, le soutien émotionnel, l'aide tangible).

Tableau 2

Types de soutien et nature de l'aide apportée

Auteurs dans Tardy, 1985)

Appréciation
Informationnel

\section{Types de soutien}

Émotionnel

Instrumental

\section{Nature de l'aide apportée}

Manifestation de confiance, d'empathie, d'amour, de bienveillance

Prêt d'argent, assistance technique, transport

Donner de l'information, des avis et des conseils

Rétroaction (exemple: tu fais un bon travail)

\begin{tabular}{|c|c|c|}
\hline \multirow[t]{6}{*}{ Barrera (1981) } & Aide matérielle & $\begin{array}{l}\text { Aide financière, prêt ou don } \\
\text { d'objets physiques }\end{array}$ \\
\hline & Assistance physique & Partage des tâches \\
\hline & Interaction intime & $\begin{array}{l}\text { Expression et partage } \\
\text { des sentiments et } \\
\text { des préoccupations }\end{array}$ \\
\hline & Conseil & Avis, «guidance» \\
\hline & Rétroaction & Information sur soi-même \\
\hline & Participation sociale & Activités de loisirs \\
\hline \multirow[t]{4}{*}{ Cohen et Wills (1985) } & Estime & $\begin{array}{l}\text { Manifestation d'affection, } \\
\text { de soutien émotionnel, } \\
\text { de sympathie } \\
\text { et d'encouragement }\end{array}$ \\
\hline & Informationnel & $\begin{array}{l}\text { Aide dans la définition } \\
\text { et la compréhension } \\
\text { d'événements probléma- } \\
\text { tiques; éducation, conseils, } \\
\text { références }\end{array}$ \\
\hline & Accompagnement social & $\begin{array}{l}\text { Activités de loisirs } \\
\text { et activités sociales }\end{array}$ \\
\hline & Instrumental & Aide financière et matérielle \\
\hline
\end{tabular}




\section{LES INSTRUMENTS DE MESURE DU SOUTIEN SOCIAL}

Cette troisième partie du présent article est consacrée à la description de certains instruments de mesure du soutien social. La recension réalisée, bien que non exhaustive, a permis de relever plus d'une quarantaine d'instruments construits dans le but de mesurer le soutien social. Afin de respecter les limites inhérentes à la rédaction de cet article, il fut convenu de retenir neuf instruments de mesure. Le choix de ces instruments s'est basé sur la qualité métrologique de l'instrument, de même que sur la représentativité des trois principales dimensions du soutien social, soit les ressources du réseau de soutien, les comportements de soutien et l'appréciation subjective du soutien. Les instruments n'ayant subi aucune épreuve de fiabilité ou de validité n'ont pas été retenus.

Pour chacun des neuf instruments retenus, nous présenterons la dimension du soutien social mesurée, une description sommaire et, enfin, les résultats obtenus dans le cadre d'épreuves réalisées pour en assurer la fiabilité et la validité.

\section{Inventory of Socially Supportive Behaviors (ISSB)}

Élaboré par Barrera, Sandler et Ramsey (1981), le Inventory of Socially Supportive Behaviors (ISSB) mesure les comportements qui procurent du soutien à un individu. L'instrument comporte 40 énoncés pour lesquels le répondant doit indiquer, à l'aide d'une échelle intervallaire de cinq points allant de "pas du tout» à «environ tous les jours», la fréquence du comportement décrit dans l'énoncé, au cours des quatre dernières semaines. Par exemple, le répondant doit indiquer, pour les quatre dernières semaines, «le nombre de fois où une personne lui a démontré qu'il faisait quelque chose de bien", ou encore «le nombre de fois où une personne lui a assuré un moyen de transport». L'instrument mesure des comportements d'aide tangibles et non tangibles.

Les concepteurs de l'ISSB ont évalué les qualités psychométriques de leur instrument auprès d'un échantillon de 71 étudiants universitaires. Les résultats ont démontré une fidélité test-retest de 0,88 (intervalle de deux semaines). La consistance interne fut évaluée à partir du coefficient alpha de Cronbach et celle-ci varie de 0,92 à 0,94 selon les différentes souséchelles. À l'aide d'un deuxième échantillon $(\mathrm{N}=55)$, Barrera et ses collaborateurs ont démontré que la fréquence des interactions supportantes, telle qu'elle est mesurée avec le ISSB, était 
significativement reliée avec la grandeur du réseau social (c'està-dire le nombre de personnes), mesurée à l'aide du Arizona Social Support Interview Schedule (instrument décrit ci-après). Enfin, ces auteurs rapportent une relation positive entre la fréquence des interactions supportantes des membres de la famille et la perception de la cohésion familiale (Barrera et al., 1981).

\section{Arizona Social Support Interview Schedule (ASSIS)}

Le Arizona Social Support Interview Schedule (ASSIS) (Barrera, 1981) a été élaboré à la suite du ISSB, afin d'évaluer le réseau de soutien social et la satisfaction du sujet à l'égard du soutien reçu. L'ASSIS permet d'identifier les personnes qui fournissent les types de soutien suivants: aide matérielle (aide financière ou autres objets physiques), assistance physique (partage de tâches), interaction intime (expression de sentiments et de soucis personnels), conseils (avis, guide), rétroaction (information sur soi-même) et participation sociale (loisirs). Pour chacun de ces six types de soutien, on demande au répondant d'identifier les personnes qui seraient susceptibles d'apporter une aide (ex.: «Si vous aviez besoin de quelque chose de matériel, quelles personnes pourraient vous aider?»). On demande ensuite au répondant s'il a reçu ce type de soutien au cours du dernier mois; dans l'affirmative, il doit indiquer son degré de satisfaction relatif à ce soutien. Il est aussi demandé au répondant d'identifier les personnes avec lesquelles il a eu des interactions conflictuelles (ou non plaisantes). Finalement, les répondants doivent donner certaines informations sur les caractéristiques des membres du réseau (conjoint, parents, fratrie, amis, voisins, enseignant, médecin, conseillers, etc.).

Les qualités métrologiques de l'ASSIS ont été vérifiées par Barrera et ses collaborateurs auprès d'un échantillon de 45 étudiants universitaires. Les résultats ont démontré une fidélité variable selon les différentes sous-échelles. Ainsi, les résultats des analyses test-retest (intervalle de deux jours) sont: 0,88 pour $l a$ grandeur du réseau; 0,54 pour la présence de relations conflictuelles; 0,69 pour la satisfaction du soutien reçu; 0,80 pour le besoin de soutien. La consistance interne de chacune de ces sous-échelles fut évaluée à partir du coefficient alpha de Cronbach. Les résultats suggèrent des valeurs non consistantes variant de 0,33 à 0,70 , selon les sous-échelles.

L'ASSIS a été traduit en français et validé auprès d'une population québécoise (Lepage, 1984, données rapportées par 
Séguin et al., 1993). Les résultats de ces analyses (coefficients alpha de Cronbach) ont démontré une consistance interne plus élevée pour l'ensemble des sous-échelles de l'ASSIS alors que les valeurs obtenues variaient de 0,81 à 0,86 .

\section{Interpersonal Support Evaluation List (ISEL)}

Le Interpersonal Support Evaluation List (ISEL) a été élaboré par Cohen, Mermelstein, Karmack et Hoberman (1985). Le ISEL consiste en une liste de 40 énoncés concernant la perception de la disponibilité de ressources sociales. Ces énoncés sont répartis en quatre sous-échelles qui mesurent des types de soutien différents: l'aide matérielle, l'appréciation, l'estime de soi et le sentiment d'appartenance. La moitié des items sont formulés positivement (ex. : "Il y a plusieurs personnes différentes avec qui il est plaisant d'être») et l'autre moitié négativement (ex.: "Je sens qu'il n'y a personne avec qui je peux discuter de mes inquiétudes et de mes peurs»). On demande au répondant de dire pour chaque énoncé si cela est "probablement vrai»ou «probablement faux».

Cohen et ses collaborateurs (1985) décrivent les qualités métrologiques du ISEL en rapportant les données tirées de 12 études dont le nombre de participants varie entre 31 et 330 personnes. Les résultats des analyses test-retest fluctuent entre 0,63 et 0,87 selon les études. La consistance interne pour l'échelle totale et pour chacune de ces sous-échelles a été évaluée à partir du coefficient alpha de Cronbach. Selon les études, l'échelle totale affiche des scores variant entre 0,77 et 0,90, alors que pour les sous-échelles les scores varient de la façon suivante: l'appréciation, de 0,70 à 0,92, l'estime de soi, de 0,60 à 0,78, le sentiment d'appartenance, de 0,73 à 0,78 , et, enfin, le soutien tangible, de 0,71 à 0,81 .

Pour ce qui est de la validité, les auteurs ont procédé à plusieurs analyses corrélationnelles entre le ISEL et quelques instruments de mesure. Ainsi, bien que ces résultats ne soient pas tous publiés, il semblerait qu'il y ait absence de corrélation entre le ISEL et la désirabilité sociale, telle que mesurée avec le CrowneMarlowe Social Desirability (Crowne et Marlowe, 1960). Par ailleurs, une corrélation de 0,46 est observée entre le ISEL et le ISSB, ce qui, selon les auteurs, serait une indication de sa validité discriminante car ces deux instruments prétendent mesurer des dimensions différentes du soutien social. Finalement, les données émanant de ces études indiquent que plus le score obtenu 
à l'aide du ISEL est élevé (donc, plus la perception de la disponibilité de soutien est élevée), plus les scores de dépression obtenus avec l'Inventaire de Beck sont faibles (les corrélations variant de $-0,38$ à $-0,60$ ).

\section{Norbeck Social Support Questionnaire (NSSQ)}

Le Norbeck Social Support Questionnaire (NSSQ) a été élaboré par Norbeck, Lindsey et Carrieri (1981), et cet instrument mesure plusieurs dimensions du soutien social, notamment les ressources du réseau de soutien et l'appréciation subjective du soutien. Les répondants sont invités à faire l'inventaire des personnes qui leur sont significatives et ce, jusqu'à un maximum de 20 individus. Pour chacune des personnes identifiées, on demande aux répondants de préciser le type de relations (ex.: conjoint, amis, connaissances, voisins ou autres) et, à l'aide de neuf questions comportant une échelle intervallaire, on les invite à se prononcer sur la qualité de ces relations. Voici un exemple de question: "Jusqu'à quel point cette personne fait-elle en sorte que vous vous sentez aimé?»

Les auteurs ont évalué les qualités métrologiques du NSSQ auprès de deux échantillons: un premier groupe composé de 75 personnes étudiant en nursing ( 74 femmes et 1 homme) et un deuxième composé de 60 étudiants «senior» en nursing (54 femmes et 6 hommes). En ce qui concerne la fidélité, les analyses rapportent des corrélations test-retest (intervalle d'une semaine) variant entre 0,71 et 0,92 . L'évaluation de la consistance interne à l'aide du coefficient alpha de Cronbach indique des scores variant entre 0,69 et 0,98 .

De plus, Norbeck et ses collaborateurs (1981) ont évalué la présence de réponses biaisées par la désirabilité sociale (CrowneMarlowe Social Desirability, Crowne et Marlowe, 1960). Les résultats de leurs analyses suggèrent des corrélations variant de 0,01 à 0,17 , ce qui invite les auteurs à conclure que leur instrument est peu sensible à l'effet de la désirabilité sociale. Par la suite, les auteurs ont étudié la validité de construit du NSSQ en le comparant avec une autre mesure du soutien social, soit le Social Support Questionnaire élaboré par Cohen et Lazarus (1981, dans Norbeck et al., 1981). Les corrélations entre les différentes dimensions mesurées variaient de $-0,03$ à 0,56 . D'autre part, Norbeck et ses collaborateurs (1981) rapportent des résultats non significatifs lorsque des scores obtenus à l'aide du NSSQ sont mis en relation avec des scores obtenus pour les mêmes sujets à l'aide 
du Profile of Mood States (McNair, Lorr et Droppleman, 1971), un instrument qui mesure certains symptômes psychiatriques. Toujours dans le but de vérifier la validité de construit du NSSQ, Norbeck et ses collaborateurs ont entrepris une deuxième étude (Norbeck, Lindsey et Carrieri, 1982). Leur conclusion est que les scores obtenus à l'aide du NSSQ présentent des indices de corrélation satisfaisants avec des scores provenant du Fundamental Interpersonal Relations Orientation (Schutz, 1978), qui évalue des dimensions associées aux relations interpersonnelles analogues à celles ciblées par le NSSQ.

\section{Perceived Social Support from Friends and From Family (PSS-Fr et PSS-Fa)}

Le Perceived Social Support from Friends and from Family (PSS-Fr et PSS-Fa) a été élaboré par Procidano et Heller (1983), afin de mesurer comment les besoins de soutien, d'information et de rétroaction sont comblés par les amis (PSS-Fr) et par la famille (PSS-Fa). Cette évaluation repose sur la perception du soutien reçu. L'instrument contient quarante énoncés, dont vingt portent sur le soutien apporté par les amis (PSS-Fr) et vingt, par les membres de la famille (PSS-Fa). Les répondants doivent indiquer «oui » ou «non», «ne sais pas» pour chacun des énoncés (exemple d'énoncé pour les amis: "Mes amis me soutiennent moralement lorsque j'en ai besoin", pour la famille: "Ma famille est sensible à mes besoins personnels »).

La validité de l'instrument a été vérifiée auprès de trois échantillons composés d'un nombre de participants variant entre 105 et 222 personnes. En ce qui concerne la fidélité, les résultats indiquent une corrélation test-retest de 0,83 et la consistance interne, évaluée à l'aide du coefficient alpha de Cronbach, est de 0,88 pour le PSS-Fr et de 0,90 pour le PSS-Fa. Enfin, une analyse factorielle indique que chaque échelle est composée d'un seul facteur.

Pour évaluer la validité, les auteurs ont mis le PSS en relation avec d'autres instruments. Ainsi, les symptômes de psychopathologie (évalués avec le Short Form MMPI, Faschinghauer, 1974) sont moins présents chez les personnes qui perçoivent avoir un bon soutien, tel qu'il est mesuré avec le PSS. La version pour les amis est plus fortement reliée à la compétence sociale (mesurée avec le Dating and Assertion Questionnaire, Levenson et Gottman, 1978). De plus, l'humeur négative (mesurée avec le Multiple Affect Adjective Checklist, Zuckerman, 1960) des 
répondants est en lien avec la perception de recevoir un faible soutien de la part des amis, tandis qu'une appréciation élevée de soutien de la part de ces derniers est en relation avec une basse anxiété. Finalement, Procidano et Heller (1983) rapportent également que les sujets qui perçoivent avoir un bon soutien de la part des amis parlent davantage d'eux-mêmes que les sujets ayant la perception de recevoir un moins bon soutien de la part de leurs amis.

Cet instrument de mesure a été traduit en français et validé auprès d'une population québécoise (Vézina, 1988). Les résultats indiquent un coefficient alpha de 0,87 pour chacune des échelles (famille et amis).

\section{Social Support Questionnaire (SSQ)}

Élaboré par Sarason, Levine, Basham et Sarason (1983), le Social Support Questionnaire (SSQ) est un instrument qui propose d'évaluer la perception de la disponibilité et de la satisfaction du soutien social. L'administration du SSQ se réalise en deux étapes. Dans la première, les répondants doivent indiquer le nom ou les initiales des personnes qui leur apportent du soutien et ce, pour chacun des 27 énoncés contenus dans le questionnaire (ex.: "Sur qui pouvez-vous réellement compter lorsque vous avez besoin de parler?»). On établit ainsi la liste des personnes du réseau. Dans une deuxième partie, les répondants indiquent pour chaque énoncé leur degré de satisfaction au regard du soutien reçu.

La fiabilité du SSQ a été vérifiée auprès d'un échantillon de 602 étudiants universitaires de premier cycle (Sarason et al., 1983). Les corrélations test-retest (intervalle de 4 semaines) sont de 0,90 pour le nombre de personnes identifiées et de 0,83 pour la satisfaction du soutien reçu. La consistance interne est de 0,97 pour le nombre de personnes identifiées et de 0,94 pour la satisfaction du soutien reçu. La corrélation entre le nombre de personnes identifiées et le degré de satisfaction est de 0,34. Selon Sarason et ses collaborateurs, cette corrélation indique que les deux dimensions sont différentes et que, en somme, la perception de la disponibilité des ressources du réseau de soutien, reflétée par le score $\mathrm{N}$, et la satisfaction envers le soutien qui est disponible, reflétée par le score $S$, méritent d'être appréciées distinctement dans l'analyse. Elle incite également ces auteurs à remettre en question la vision globalisante du concept de soutien social car, selon eux, une telle globalité devrait se 
traduire par un indice de corrélation plus élevé entre les deux dimensions ainsi mesurées.

En ce qui a trait à la validité, plusieurs études de type corrélationnel ont été réalisées en utilisant le SSQ et divers instruments psychométriques (données rapportées dans Sarason et al., 1983). Les résultats de ces études suggèrent une corrélation négative $(-0,39$ à -0,24), entre le SSQ et le Multiple Affect Adjective Check List (Zuckerman, 1960), un instrument qui mesure l'anxiété, la dépression et l'hostilité. Ainsi, la disponibilité d'un bon soutien serait associée à des indices faibles d'anxiété, d'hostilité et de dépression. Par ailleurs, les personnes qui bénéficient d'un bon soutien social seraient plus optimistes quant à leur avenir. Finalement, Sarason et ses collaborateurs démontrent que le SSQ serait peu sensible à l'effet de la désirabilité sociale (mesurée avec le Crowne-Marlow Social Desirability, Crowne et Marlowe, 1960).

\section{Social Support Appraisals Scale (SS-A)}

Le Social Support Appraisals Scale (SS-A) a été élaboré par Vaux en 1982 (voir Vaux et al., 1986); cet instrument fait partie d'une batterie de tests comprenant trois instruments développés par Vaux en 1982. Chaque instrument mesure une dimension différente du soutien social. Le SS-A mesure l'appréciation subjective du soutien social. Il comporte 23 items qui mesurent la perception qu'entretient une personne à propos de l'amour, de l'affection et enfin de l'estime que lui apportent ses proches. L'instrument s'intéresse également à la mesure du degré d'engagement de la personne au sein de son réseau affectif et social. Le répondant indique s'il est totalement d'accord, en accord, en désaccord ou totalement en désaccord avec les 23 énoncés soumis à son attention (exemples d'énoncés: "Mes amis me respectent», "Ma famille me tient en estime»). Trois scores sont obtenus: le SS-A total (la somme des 23 items), le SS-A famille (la somme des 8 items reliés à la famille) et le SS-A amis (la somme des 7 items reliés aux amis). Les huit items restants correspondent aux autres personnes appartenant au réseau socio-affectif du sujet. Le type de soutien mesuré est essentiellement le soutien affectif.

Le SS-A a été vérifié auprès de 10 échantillons différents dont le nombre de sujets varie entre 44 et 156 (Vaux et al., 1986). La fidélité se traduit par des indices de corrélation test-retest variant entre 0,71 et 0,80 (Vaux, 1992). La consistance 
interne de l'instrument, évaluée à l'aide du coefficient alpha de Cronbach, est établie à 0,90 pour le SS-A total, à 0,80 pour la sous-échelle SS-A famille et à 0,84 pour la sous-échelle SS-A amis. Les corrélations inter-échelles (amis $v s$ famille) indiquent des valeurs variant entre 0,51 et 0,52 , ce qui semble justifier la nécessité d'avoir recours à ces deux sous-échelles.

En ce qui a trait à la validité de l'instrument, Vaux et ses collaborateurs (1986) rapportent, entre autres, des données corrélationnelles entre le SS-A et divers instruments qui mesurent l'état de détresse et de bien-être. Ainsi, des corrélations variant entre $-0,27$ et $-0,55$ sont présentes entre l'humeur dépressive (mesurée avec le Center for Epidemiological Studies Depression Scale, Radloff, 1977) et les résultats au SS-A. De plus, il existe une relation négative (de -0,40 à-0,71) entre le SS-A et une échelle qui mesure la solitude (UCLA Revised Loneliness Scale, Shaul, 1981).

\section{Social Support Behaviors Scale (SS-B)}

Le Social Support Behaviors Scale (SS-B) a été élaboré par Vaux en 1982 (voir Vaux, Riedel et Stewart, 1987). Il a été conçu afin de mesurer cinq types de comportements de soutien, soit le soutien émotionnel, l'assistance pratique, l'assistance financière, la socialisation et les conseils/avis. Les comportements considérés proviennent de deux sources, soit la famille et les amis. Le SS-B comporte 45 énoncés qui décrivent des comportements de soutien (ex.: "Aller voir un film», «Prêter de l'argent»). Le répondant doit alors indiquer sur une échelle de cinq intervalles le degré de réalisation, par un membre de la famille ou par les amis, du comportement de soutien décrit dans l'énoncé.

Les informations sur la fidélité de l'instrument ne sont pas connues, alors que sa consistance interne, évaluée à l'aide du coefficient alpha de Cronbach, est de 0,85 . En ce qui a trait à la validité de l'instrument, Vaux et ses collaborateurs observent, à partir d'un échantillon de 120 étudiants, des corrélations modérées entre le SS-B et le ISSB de Barrera (de -0,17 à 0,42). De plus, les résultats d'une analyse factorielle indiquent une correspondance entre les items du SS-B et les cinq types de comportements opérationnalisés, soit le soutien émotionnel, l'assistance pratique, l'assistance financière, la socialisation et les conseils/avis (Vaux et al., 1987). 


\section{Social Support Resources Scale (SS-R)}

Le Social Support Resources Scale (SS-R) a également été élaboré en 1982 par Vaux (voir Vaux et Harrison, 1985). Le SS-R est un instrument bâti afin de mesurer les ressources du réseau de soutien. Le répondant est invité à dresser une liste de 10 personnes qui lui apportent de l'aide selon les cinq types de soutien suivants: soutien émotionnel, assistance pratique, assistance financière, socialisation et conseils/avis. Cela permet l'identification d'une cinquantaine de personnes au maximum. Chaque type de soutien est décrit et des questions spécifiques sont posées pour faciliter le rappel. Par exemple, les questions accompagnant la description du soutien émotionnel sont: "Quelles sont les personnes qui vous soutiennent lorsque vous avez du chagrin?», «À qui vous confiez-vous?», «Avec qui discutez-vous de sentiments personnels?». On obtient ainsi un score qui reflète la grandeur totale du réseau (excluant les répétitions de noms entre chaque type) et cinq scores spécifiques en fonction du type (permettant les répétitions de noms entre les cinq types). Le répondant complète alors les items élaborés pour évaluer divers aspects de la relation, tels que la fréquence des interactions, la proximité, la réciprocité de la relation, la complexité, la nature de la relation (conjoint, amis, etc.), les caractéristiques environnementales (classe d'école, voisin) et le nombre de membres du réseau que chaque membre connaît (la densité).

Le Social Support Resources Scale (SS-R) a été vérifié auprès d'un échantillon de 98 personnes. La fidélité se traduit par des indices de corrélation test-retest variant de 0,46 à 0,72 selon l'aspect mesuré (intervalle de six semaines). La consistance interne du SS-R évaluée à l'aide du coefficient alpha de Cronbach est de 0,76 . Vaux et ses collaborateurs rapportent des corrélations modestes entre le SS-R et le SS-A, ce qui est une indication du potentiel discriminant de ces instruments.

\section{DISCUSSION}

Dans la partie précédente, nous avons présenté des instruments de mesure choisis en fonction de certaines caractéristiques, soit la qualité métrologique de l'instrument et la représentativité des trois concepts de soutien social. Le but de cette présentation essentiellement descriptive était de faciliter le processus de décision quant au choix d'un instrument pour mesurer le soutien 
social dans le cadre d'une recherche. Cependant, il serait pertinent à cette étape-ci de soumettre quelques recommandations, afin de guider le choix d'un instrument.

Il est bien sûr recommandé d'utiliser un instrument dont les qualités métrologiques sont démontrées. Si les instruments décrits précédemment sont relativement bien documentés en ce qui concerne leur fidélité et leur consistance interne, il n'en est pas de même pour les différents aspects reliés à leur validité. En effet, Barrera et al. (1981) et Barrera (1981) donnent peu de renseignements sur la validité de leur instrument, tandis que Norbeck et al. (1981, 1982), Procidano et Heller (1983), Sarason et al. (1983) et Vaux et al. (1986) semblent avoir bien évalué cette propriété métrologique au regard des instruments qu'ils proposent. Souvent de nature corrélationnelle, ces données représentent la relation entre le soutien social et d'autres instruments choisis en fonction d'un certain construit théorique. Par exemple, une relation négative entre le degré de soutien social et l'anxiété (mesurée à l'aide d'un test psychométrique) est généralement attendue. De plus, les auteurs mentionnés dans cet article ont, de façon générale, identifié les différents instruments qu'ils ont retenus pour réaliser leurs études de validité. Leurs choix à l'égard des instruments retenus sont toutefois peu justifiés. Aussi le lecteur est-il invité à prêter une attention particulière à cette question.

Un aspect incontournable dans le choix d'un instrument concerne la dimension du soutien social que l'on désire mesurer. En effet, certains instruments ne mesurent qu'une seule dimension du soutien social, tandis que d'autres proposent d'en mesurer deux, parfois trois. Bien que le présent article évoque le fait que le soutien social comporte plusieurs dimensions, il se peut qu'un projet de recherche ne s'intéresse qu'à une seule de ces dimensions. Dans un tel cas, il est essentiel de bien circonscrire la dimension à l'étude. Le projet s'intéresse-t-il aux ressources du réseau (grandeur du réseau, structure du réseau, etc.), à l'appréciation subjective du soutien (satisfaction à l'égard du soutien reçu, perception de l'adéquation du soutien, etc.) ou aux comportements dits supportants? Le fait de rendre explicites la ou les dimensions à l'étude facilitera le choix de l'instrument. Cependant, certains projets de recherche se proposent d'évaluer plusieurs dimensions du soutien social car, en soi, il s'agit d'un concept multidimensionnel. Ainsi qu'il a été démontré dans le cadre du présent article, certains instruments ont été élaborés 
afin de tenir compte des multiples dimensions du soutien social. L'ASSIS, élaboré par Barrera (1981), et les trois instruments de Vaux (1982, présentés par Vaux et al., 1986; Vaux et al., 1987; Vaux et Harrison, 1985) s'inscrivent dans cette perspective. L'ASSIS semble tenir compte des trois principales dimensions du soutien: les ressources du réseau, les comportements de soutien et l'appréciation subjective du soutien. L'instrument s'est révélé fidèle et a montré une bonne consistance interne; il est cependant peu documenté en ce qui a trait à sa validité. Par contre, il présente l'avantage d'avoir été traduit en français et validé auprès d'une population québécoise.

Vaux nous propose un ensemble de trois instruments, chacun mesurant une dimension du concept. L'utilisation de ces instruments peut également se révéler un choix intéressant dans le cadre d'une recherche qui s'intéresserait aux multiples dimensions du soutien social. En effet, ces trois instruments présentent de bonnes qualités métrologiques.

Enfin, les instruments proposés par Barrera et par Vaux offrent en plus la possibilité de documenter le type de soutien (instrumental, émotionnel, informationnel, etc.) ainsi que la source de ce soutien (famille, amis, etc.).

\section{CONCLUSION}

L'objectif du présent article était de clarifier la notion de soutien social en définissant les différents cadres conceptuels qui lui sont associés et en répertoriant les principaux instruments reconnus pour en assurer la mesure. Cette démarche a conduit à l'identification des trois principales dimensions du soutien social, soit les ressources du réseau de soutien, les comportements de soutien et l'appréciation subjective de soutien. Enfin, nous avons décrit des instruments de mesure du soutien social correspondant aux dimensions identifiées, et ce, en précisant leurs qualités métrologiques. 


\section{Références bibliographiques}

BARRERA, M., I.N. SANDLER et T.B. RAMSEY (1981). "Preliminary development of a scale of social support », American Journal of Community Psychology, no 9, p. 435-447.

BARrera, M. (1981). "Social Support in the Adjustment of Pregnant Adolescents : Assessment Issues », dans B.H. Gottlieb (dir.), Social Networks and Social Support, Beverly Hills, CA : Sage, p. 69-96.

BArrerA, M. (1986). "Distinctions Between Social Support Concepts, Measures, and Models ", American Journal of Community Psycho$\log y$, vol. 14, no 4, p. 413-445.

Blanchard, C.G., T.L. Albrecht, J.C. Ruckdeschel, C.H. Grant et R.M. HEMMICK (1995). "The Role of Social Support in Adaptation to Cancer and to Survival», Journal of Psychosocial Oncology, vol. $13, \mathrm{n}^{\circ} 1-2$, p. 75-95.

BozZINI, L., et R. TESSIER (1985). "Support social et santé", dans J. Dufresne, F. Dumont et Y. Martin, Traité d'anthropologie médicale. L'Institution de la santé et de la maladie, Québec: Presses de l'Université du Québec, IQRC; Lyon: Presses universitaires de Lyon.

BUCHANAN, J. (1995). "Social Support and Schizophrenia: A Review of the Literature », Archives of Psychiatric Nursing, vol. 9, $\mathrm{n}^{\circ}$ 2, p. 68-76.

Bruhn, J.G. (1991). «People Need People. Perspectives on the Meaning and Measurement of Social Support ", Integrative Physiological and Behavioral Science, vol. 26, no 4, p. 325-329.

Cohen, C., et S.L. Syme (1985). Social Support and Health, Academic Press Inc., $390 \mathrm{p}$.

CoHEN, S., et T.A. WILLS (1985). "Stress, Social Support, and the Buffering Hypothesis », Psychological Bulletin, vol. 98, n 2, p. 310-357.

Cohen, S., R. Mermelstein, T. Kamarck et H.N. Hoberman (1985). "Measuring the Functional Components of Social Support", dans I. Sarason et B. Sarason (dir.), Social Support : Theory, Research, and Applications, Dordrecht, Netherlands: Martinus Nijhoff, p. 73-94.

Ducharme, F., B. STevens et K. Rowat (1994). "Social Support: Conceptual and Methodological Issues for Research in Mental Health Nursing », Issues in Mental Health Nursing, $\mathrm{n}^{\circ}$ 15, p. 373-392.

HEITZMANN, C.A. et R.M. KAPLAN (1988). «Assessment of Methods for Measuring Social Support», Health Psychology, vol. 7, n 1, p. 75-109.

House, J.S., R.L. KAHN, J.D. MCLEOD et D. Williams (1985). « Measures and Concepts of Social Support», dans S. Cohen et S.L. Syme (dir.), Social Support and Health, Academic Press Inc., p. 83-108.

NorbeCK, J.S., A.M. LindSEY et V.L. CARRIERI (1981). «The Development of an Instrument to Measure Social Support», Nursing Research, $\mathrm{n}^{\circ} 30$, p. 264-269.

NorbeCK, J.S., A.M. LindSEY et V.L. CARRIERI (1982). «Further Development of the Norbeck Social Support Questionnaire: Normative Data and Validity Testing », Nursing Research, vol. 32, $\mathrm{n}^{\circ} 1$, p. 4-9. 
O'Reilly, P. (1988). "Methodological Issues in Social Support and Social Network Research», Social Science and Medicine, vol. 26, $\mathrm{n}^{\circ} 8$, p. 863-873.

PierCe, G.R., I.G. SARASON et B.R. SARASON (1996). "Coping and Social Support", dans M. Zeider et N.S. Endler (dir.), Handbook of Coping: Theory, Research, Applications, New York: John Wiley \& Sons, p. 434-451.

Procidano, M.E., et K. Heller (1983). «Measures of Perceived Social Support from Friends and from Family: Three validation studies », American Journal of Community Psychology, vol. 11, $\mathrm{n}^{\circ} 1, \mathrm{p} .1-24$.

RiChMAN, J.M., L.B. Rosenfeld et C.J. HARDy (1993). «The Social Support Survey: A Validation Study of a Clinical Measure of the Social Support Process », Research on Social Work Practice, vol. 3, $\mathrm{n}^{\circ} 3$, p. 288-311.

RoCK, D.L., K.E. GREEN, B.K. WISE et R.D. RoCK (1984). «Social Support and Social Network Scales: A Psychometric Review», Research in Nursing and Health, $\mathrm{n}^{\circ}$ 7, p. 325-332.

SARASON, I.G., H.M. LEVINE, R.B. BASHAM et B.R. SARASON (1983). "Assessing Social Support: The Social Support Questionnaire», Journal of Personality and Social Psychology, vol. 44, $\mathrm{n}^{\circ} 1$, p. 127139.

SARASON, B.R., E.N. SHEARIN, G.R. PIERCE et I.G. SARASON (1987). «Interrelations of Social Support Measures: Theoretical and Practical Implications », Journal of Personality and Social Psychology, vol. 52, $\mathrm{n}^{\circ} 4$, p. 813-832.

SÉGuin, L., M. ST-DENis, J. Loiselle et L. PotVIn (1993). Stresseurs, soutien social et réactions dépressives à la période périnatale chez les primipares défavorisées et favorisées, Groupe de recherche interdisciplinaire en santé, Université de Montréal, 243 p.

Streeter, C.L., et C. FrankLin (1992). "Defining and Measuring Social Support : Guidelines for Social Work Practitioners », Research in Social Work Practice, vol. 2, n ${ }^{\circ}$ 1, p. 81-98.

TARDY, C.H. (1985). "Social Support Measurement», American Journal of Community Psychology, vol. 13, $\mathrm{n}^{\circ} 2$, p. 187-203.

TOUSIGNANT, M. (1988). «Soutien social et santé mentale : une revue de la littérature », Sciences sociales et santé, vol. 6, no 1, p. 77-106.

TrACy, E.M., et N. ABELL (1994). «Social network map : Some further refinements on administration ", Social Work Research, vol. 18, $\mathrm{n}^{\mathrm{o}} 1$, p. 56-60.

VAUX, A., et D. HARRISON (1985). «Social Network Characteristics Associated with Satisfaction and Perceived Support ", American Journal of Community Psychology, $\mathrm{n}^{\circ} 13$, p. 256-268.

Vaux, A., J. Phillips, L. Holly, B. Thomson, D. Williams et D. STEWART (1986). "The Social Support Appraisals (SS-A) Scale : Studies of Reliability and Validity ", American Journal of Community Psycho$\log y, 14$, p. 195-219.

VAuX, A., S. Riedel et D. STewarT (1987). "Modes of Social Support: The Social Support Behaviors (SS-B) Scale », American Journal of Community Psychology, no 15, p. 209-237. 
VAUX, A. (1988). Social Support. Theory, Research, and Intervention, New York: Praeger.

VAuX, A. (1992). «Assessment of Social Support», dans H.O.F. Veiel et U. Baumann (dir.), The Meaning and Measurement of Social Support, New York: Hemisphere Publishing Corporation, p. 193-216.

VÉZINA, A. (1988). Le travail et le réseau de support comme facteurs d'adaptation chez les veuves d'âge moyen. Thèse de doctorat. Québec: École de psychologie, Université Laval.

Weinert, C., et V.P. Tilden (1990). "Measures of Social Support: Assessment of Validity », Nursing Research, vol. 39, n ${ }^{\circ} 4$, p. 212216.

Wellman, B. (1981). "Applying Network Analysis to the Study of Support », dans B.H. Gottlieb (dir.), Social Networks and Social Support, Beverly Hills, CA : Sage, p. 171-200.

Winemiller, D.R., M.E. Mitchell, J. Sutliff et D.J. Cline (1993). "Measurement Strategies in Social Support: A Descriptive Review of the Literature », Journal of Clinical Psychology, vol. 49, $\mathrm{n}^{\circ} 5$, p. 638-648. 